\title{
Assessment of tumor necrosis factor alpha (TNF $\alpha$ ) and interleukin 6 level in gingival crevicular fluid during orthodontic tooth movement: a randomized split-mouth clinical trial
}

Parviz Padisar ${ }^{1}$, Roya Hashemi ${ }^{2}$, Mohammadreza Naseh ${ }^{3}$, Bahareh Abde Nikfarjam ${ }^{4}$, Mahdi Mohammadi ${ }^{5}$

${ }^{1}$ DMD, Associate Professor, Department of Orthodontic, Dental Faculty, Qazvin University of Medical Sciences, Qazvin, Iran

${ }^{2}$ DMD, Assistant Professor, Department of Orthodontic, Dental Faculty, Qazvin University of Medical Sciences, Qazvin, Iran

${ }^{3}$ DMD, Assistant Professor, Department of Periodontology, Dental Faculty, Qazvin University of Medical Sciences, Qazvin, Iran

${ }^{4}$ Ph.D. of Immunology, Assistant Professor, Department of Immunology, Faculty of Science, Qazvin University of Medical Sciences, Qazvin, Iran

${ }^{5}$ Ph.D. of Biostatistics, Health Promotion Research Center, School of Public Health, Zahedan University of Medical Sciences, Zahedan, Iran

Type of article: Original

\begin{abstract}
Background: Orthodontic tooth movement (OTM) is based on induction of periodontal tissue remodeling. Mechanical tooth stimulation results in the release of pro-inflammatory mediators. These mediators cause bone resorption and deposition at the pressure and tension sites and play a role in OTM. Thus, assessment of chemical biomarkers can help determine the exact amount of load and its duration of application required for each tooth and select the most efficient treatment plan with minimal complications.

Objective: This study aimed to determine the level of tumor necrosis factor alpha (TNF $\alpha$ ) and interleukin 6 (IL6) in gingival crevicular fluid (GCF) during OTM.

Methods: This randomized split-mouth clinical trial (parallel) was performed on 10 patients who were presenting to the Orthodontic Department of Qazvin University of Medical Sciences from November 2015 to June 2016. A canine tooth was randomly selected as the study group and subjected to distalization force while the contralateral canine tooth served as control. Using paper strip, GCF was collected from the study and control teeth prior to orthodontic force application (T0), one hour after (T1) and 28 days after force application (T2), then the level of TNF $\alpha$ and IL-6 was measured using ELISA. Data were analyzed using SPSS version 20 via Friedman and Wilcoxon test, and considering the significance level at $\mathrm{p}<0.05$.

Results: The level of TNF $\alpha(p=0.0799)$ and IL- $6(p=0.678)$ at both sides of study teeth was higher than both side of control teeth at T1. Also, the level of IL-6 $(\mathrm{p}=0.515)$ and $\mathrm{TNF} \alpha(\mathrm{p}=0.508)$ were higher at the tension side compared to the pressure side; but the difference was not statistically significant.

Conclusion: Due to the free circulation of GCF in gingival sulcus, the level of mediators in the GCF collected from the mesial and distal areas alone cannot serve as a suitable index for assessment of activity at the tension and pressure sites.

Trial registration: The trial was registered at the Iranian Registry of Clinical Trials with the IRCT ID: IRCT2017030632903N2.

Funding: The present study was supported by a grant from the Research Council, Qazvin University of Medical Sciences, Qazvin, Iran (thesis no.: 40).
\end{abstract}

\section{Corresponding author:}

Dr. Roya Hashemi, Department of Orthodontic, Dental Faculty, Qazvin University of Medical Science, Qazvin, Iran. Tel: +98.5433429486, Email: roya.3816@yahoo.com

Received: January 27, 2018, Accepted: June 14, 2018, Published: August 2018

iThenticate screening: May 29, 2018, English editing: July 12, 2018, Quality control: July 15, 2018

This article has been reviewed / commented by four experts

Funding / research project approval: Qazvin University of Medical Sciences (Thesis no.: 40)

Ethics approval: Qazvin University of Medical Sciences (Ref: IR.QUMS.REC.1394.812)

(C) 2018 The Authors. This is an open access article under the terms of the Creative Commons Attribution-NonCommercialNoDerivs License, which permits use and distribution in any medium, provided the original work is properly cited, the use is non-commercial and no modifications or adaptations are made. 


\section{Keywords: Gingival crevicular fluid; Interleukin-6; Tumor necrosis factor alpha; Tooth movement, Orthodontic}

\section{Introduction}

Orthodontic tooth movement is based on induction of periodontal ligament (PDL) and alveolar bone remodeling (1). Mechanical loads affect orthodontic tooth movement (OTM) via biological cell responses in the PDL, alveolar bone and tooth supporting structures $(2,3)$. Several cytokines and hormones are involved in this process (2). In general, cytokines are categorized into pro-inflammatory and anti-inflammatory cytokines (4). The pro-inflammatory cytokines include Interleukin $1 \mathrm{~b}$ (IL-1 b), IL-2, IL-5, IL-6, IL-8, interferon $\gamma$ (IFN $\gamma$ ), tumor necrosis factor alpha $(\mathrm{TNF} \alpha)$ and granulocyte macrophage colony stimulating factor (GM-CSF), which induce classic inflammation markers (5). Pro-inflammatory cytokines act primarily at the onset of orthodontic tooth movement by inducing vasodilatation and increasing vascular permeability and inflammatory response (6). These mediators cause bone resorption and deposition at the pressure and tension sites (7). IL-6 is a multifunctional cytokine (8). It not only plays a role in the immune system, but is also involved in regenerative processes and regulation of metabolism, maintenance of bone homeostasis and many neural functions (9). IL-6 promotes osteoclast differentiation (10). During orthodontic treatment, clinical and microbiological changes may be related to the concentration of cytokines particularly IL-6 and IL-8 (11). The level of IL-6 often increases within 24 hours after OTM (12). TNF $\alpha$ is a typical mediator of inflammatory responses, which is involved in the process of bone resorption (13). The level of TNF $\alpha$ increases in gingival crevicular fluid (GCF) during OTM and it induces bone resorption at the pressure site (14). GCF is a biological exudate, and measuring its components is a current method for identifying specific biomarkers with reasonable sensitivity (15). A study by Garlet showed higher expression of TNF $\alpha$, receptor activator of nuclear factor-kappaB ligand (RANKL), and matrix metalloproteinase-1 (MMP-1), in the compression side whereas the tension side presented higher expression of IL-10, tissue inhibitor of metalloproteinase-1 (TIMP-1), collagen type 1 (COL-I), osteoprotegerin (OPG) (16). However, Grant reported that tension sites exhibited significant increases in IL-1 beta, IL-8, and TNF $\alpha$ following force application, while compression sites showed increases in IL-1 beta and IL-8 after 4 hours. These data indicate that high levels of pro-inflammatory cytokines and biomarkers of tissue and bone metabolism in GCF are associated with orthodontic force application (5). Knowledge about chemical biomarkers, which indicate changes in bone remodeling, inflammation and root resorption, can help determine the exact amount of load and its duration of application required for each tooth, select the most efficient treatment plan with minimal complications and accelerate the treatment course (17). Most studies $(18,13)$ did not compare the level of cytokines at the tensile and pressure side, and in some studies $(5,16)$ both sides were compared, but these studies reported contradictory results. Also, most studies $(18,19)$ examined the cytokines level one day after force application. Thus, this study aimed to assess the level of IL- 6 and TNF $\alpha$ and compare their levels at the pressure and tension sites shortly (one hour) after force application.

\section{Material and Methods}

\subsection{Trial design}

This split-mouth blind randomized clinical trial (parallel) was performed on patients who were presenting to the Orthodontic Department of the School of Dentistry, Qazvin University of Medical Sciences from November 2015 to June 2016and were candidates for extraction of maxillary first premolars. Patients were between 10 and 21 years.

\subsection{Participants}

Patients presented to the Orthodontic Department of the School of Dentistry, Qazvin University of Medical Sciences from November 2015 to June 2016 were selected based on inclusion criteria. They were candidates for extraction of maxillary first premolars. In each patient, a canine tooth was randomly selected as the study group and subjected to distalization force $(150 \mathrm{~g})$ with coil spring while the contralateral canine tooth served as control (without force application).

\subsection{Inclusion and exclusion criteria}

The inclusion criteria were as follows: (I) systemic health, (II) no current smoking or tobacco use, (III) no intake of antibiotics, immunosuppressant, drugs changing the hormonal balance such as contraceptives, antihistamines or nonsteroidal anti-inflammatory drugs in the past four months (prior to study onset), (IV) requiring extraction of maxillary first premolars and distalization of maxillary canine teeth by fixed orthodontic treatment, (V) pocket depth (distance between sulcus depth and the most apical part of gingival margin) less than $3 \mathrm{~mm}$ on oral clinical examination, (VI) clinical attachment loss (distance between sulcus depth and cementoenamel junction) less than 2 $\mathrm{mm}$, (VII) bleeding on probing $<20 \%$ and (VIII) no radiographic evidence of alveolar bone loss. The exclusion criteria were smoking or tobacco use and intake of immunosuppressant, hormonal medications, antihistamines or non-steroidal anti-inflammatory drugs. 


\subsection{Sample size}

Ten patients including six females and four males were selected based on the inclusion criteria. Patients were selected via convenience sampling. For sample size determination, type I $(\alpha)$ and II error $(\beta)$ were taken as $5 \%$. TNF $\alpha$ at baseline and 7 days post-intervention were 0.02 and 0.07 , respectively based on the literature (4). The standard deviation for differences between the baseline of TNF $\alpha$ and 7 days post-intervention was taken as 0.04 and it was also expected to reach 0.05 difference in TNF $\alpha$ after intervention.

\subsection{Randomization and blinding}

In this study, we used permuted block randomization method for selection of canine tooth at one side as the study group, and the contralateral canine as the control group, in any patient (split mouth). Samples were coded and the immunologist was blinded to the group allocation of samples (Figure1).

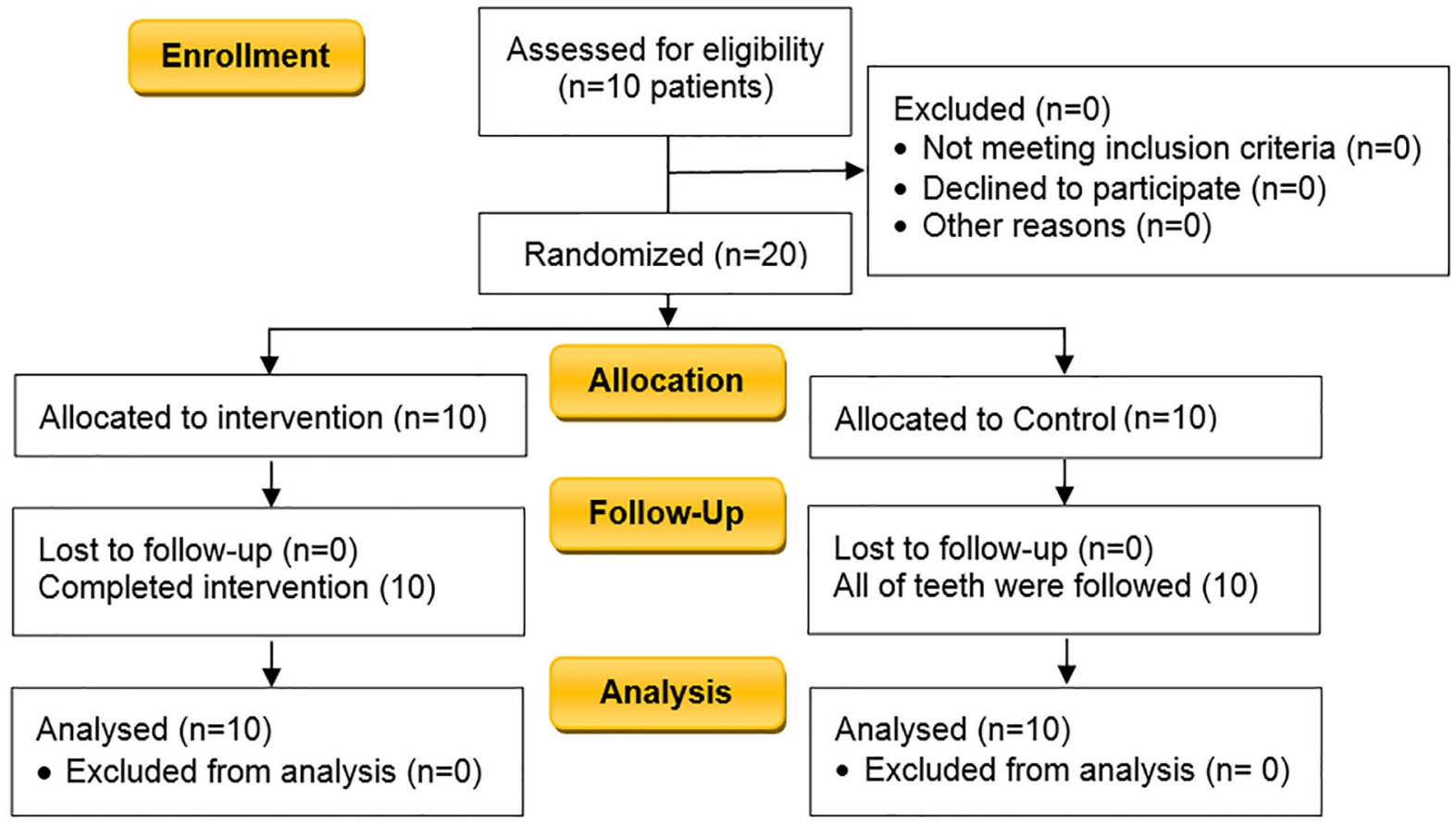

Figure 1. Consort-2010 Flow Diagram of the study

\subsection{Outcomes}

Level of proinflammatory cytokines (IL-6, TNF $\alpha$ ) in GCF was collected by periopaper strip from the control and study teeth prior to orthodontic force application (T0), one hour (T1) and 28 days after force application (T2), and measured by ELIZA.

\subsection{Confounding factors}

After considering the inclusion and exclusion criteria, 10 patients were enrolled. The confounding factors of this study include factors that can alter the level of inflammatory cytokine, such as periodontal disease and systemic diseases, smoking or tobacco, antibiotics, immunosuppressant, drugs changing the hormonal balance, such as contraceptives, antihistamines, or non-steroidal anti-inflammatory drugs. Thus, the confounding factors were limited, based on the inclusion criteria. The study was also done by split-mouth, so there was no need for the two groups to be matched. However, age and gender were matched because in each patient, one tooth was as control and the contralateral tooth was as study group.

\subsection{Intervention and measurements}

Prior to the study, all patients underwent phase I periodontal therapy and used chlorhexidine mouth rinse for two weeks. They also received instructions on correct tooth brushing using Bass method and correct use of dental floss. 


\subsubsection{Collection of GCF before orthodontic appliance Placement}

To allow tissue healing, one week after extraction of maxillary first premolars and immediately before the bonding of orthodontic attachments, baseline GCF (T0) was collected using periopaper strip (Oraflow, NY, USA). Prior to collection of GCF, the teeth were isolated by cotton rolls. Then, gentle water and air spray was used to eliminate blood and saliva from the test and control teeth. Using periopaper strip, GCF was collected at the mesial and distal sites of the study and control teeth. The periopaper strip was inserted into the sulcus until resistance was felt and remained there for 30 seconds. In case of contamination with blood or saliva, it was discarded and the procedure was repeated. After collection of GCF, periopaper strip was sealed in sterile polypropylene containers and stored at -20 ${ }^{\circ} \mathrm{C}$ until ELISA.

\subsubsection{Placement of orthodontic appliance}

First, the upper first molar teeth were banded. Then a trans palatal arch was placed. In addition, 0.022 inch standard edgewise bracket was bonded to the second premolar and canine teeth at both study and control quadrants. The posterior teeth with stainless steel segment wire $(0.019 \times 0.025)$ were fixed passively and $0.017 \times 0.025$ titanium molybdenum alloy segment wire was used to fabricate a simple vertical loop with $7 \mathrm{~mm}$ height and $1 \mathrm{~mm}$ width. Distal to the loop, a helix was used to attach the coil spring. After the vertical loop was made in the manner described, it was placed in the axillary tube of first molar and passively tied to the control canine tooth. For the study teeth, the same loop was used as in the control tooth with the exception that a $20^{\circ}$ gable bend at each side of the loop was used to prevent tipping. Moreover, $30^{\circ}$ anti-rotation was used at the mesial of the loop. Next, $150 \mathrm{~g}$ distalizing force was only applied to the study tooth using medium NiTi closed coil spring with eyelets (G and H Company, USA). It was placed between the molar hook and loop. Figures 2 and 3 indicate placement of attachment on test and control teeth, respectively.

\subsubsection{GCF collection after orthodontic appliance placement}

GCF samples were collected again at the study and control sides at one hour (T1) and 28 days (T2) after the application of distalizing load.

\subsubsection{ELIZA}

Each tube containing paper strip was diluted with $250 \mu \mathrm{L}$ of phosphate buffered saline and centrifuged for five minutes at $\times 2000 \mathrm{~g}$ and $4{ }^{\circ} \mathrm{C}$. After removing the paper strips, the supernatant was divided into two aliquots. The samples were then examined by an immunologist using ELISA (eBioscience, NY, USA) according to the kit manufacturer's instructions for determining the level of IL-6 and TNF $\alpha$. After coloring, optical density of the solution was read at $450 \mathrm{~nm}$ wavelength by a spectrophotometer. The total volume of both cytokines was determined in pictograms (pg). The concentration of each sample was determined by dividing the amount of cytokine by the sample volume $(\mathrm{pg} / \mu \mathrm{L})$.

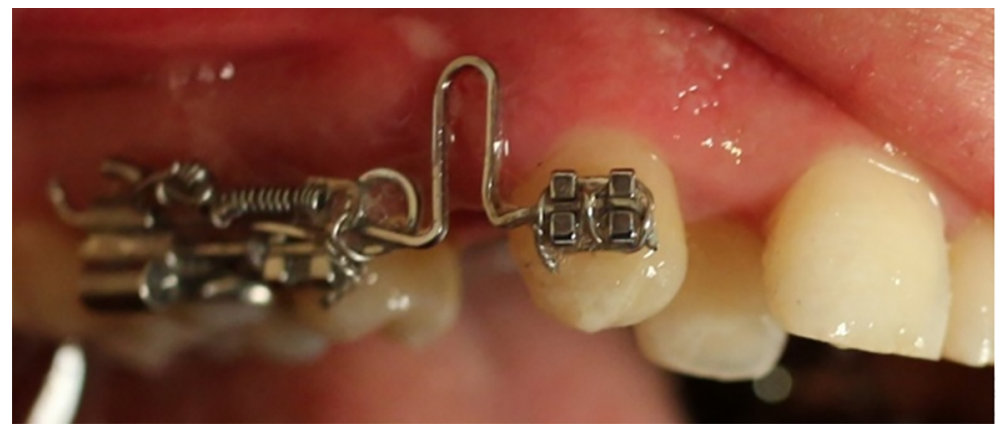

Figure 2. Test canine tooth under distalizing load applied by NiTi closed coil spring

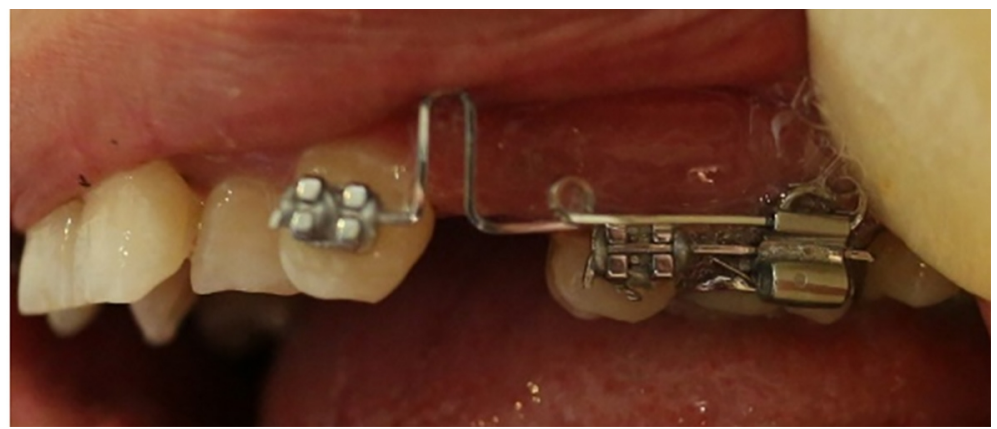




\subsection{Statistical analyses}

Figure 3. Control canine tooth without applying distalizing load

Data were analyzed using IBM@ SPSS $\odot$ Statistics version 20 (IBM@ Corp., Armonk, NY, USA). As IL-6 and $\mathrm{TNF} \alpha$ were not normally distributed based on Shapiro-Wilk test, the Friedman test was used to compare changes in level of cytokines at different time points. Wilcoxon signed rank test was used to compare the mean level of cytokines at the tension (mesial) and pressure (distal) sites of the study tooth and also for the purpose of comparison of changes in level of cytokines at the control side.

\subsection{Ethical considerations}

The study protocol was approved by the Medical Ethics Committee of Qazvin University of Medical Sciences (IR.QUMS.REC.1394.812) and this study was registered at the Iranian Registry of Clinical Trials (IRCT2017030632903N2). Before collecting the data, informed consent was obtained from all patients. It was explained to all patients that the implementation of this research does not pose any danger to them. The objectives of the study were also explained to all the participants. Then, personal information forms were completed by all the participants. The participants were ensured that they could withdraw from the study at any stage.

\section{Results}

Ten patients including six females and four males participated in this study with a mean age of $16.1 \pm 4.18$ years. All patients who had the criteria for entering the study needed orthodontic treatment, so all of these patients were enrolled in the study, and none of them left the study. This study did not have any unwanted side effects for the control and study group. The concentration of each cytokine was calculated by placing the optical density in the equation obtained from the standard curve. Table 1 shows the mean concentration of cytokines in the test and control groups at the mesial and distal sites.

Table 1. Level of IL-6 cytokine in GCF of test and control canine teeth (in pictograms per microliter)

\begin{tabular}{|c|c|c|c|c|c|}
\hline Group & Side & Measurement time & Mean & Standard deviation & Median \\
\hline \multirow[t]{6}{*}{ test } & \multirow[t]{3}{*}{ Distal (pressure) } & T0 & 2.86 & 2.36 & 1.86 \\
\hline & & $\mathrm{T} 1$ & 3.45 & 3.27 & 1.78 \\
\hline & & $\mathrm{T} 2$ & 2.68 & 2.43 & 1.54 \\
\hline & \multirow[t]{3}{*}{ Mesial (tension) } & T0 & 3.11 & 2.73 & 1.78 \\
\hline & & $\mathrm{T} 1$ & 3.51 & 3.37 & 1.52 \\
\hline & & $\mathrm{T} 2$ & 3.19 & 2.26 & 2.04 \\
\hline \multirow[t]{6}{*}{ Control } & \multirow[t]{3}{*}{ Distal (pressure) } & T0 & 2.21 & 1.49 & 1.81 \\
\hline & & $\mathrm{T} 1$ & 2.80 & 2.53 & 1.77 \\
\hline & & $\mathrm{T} 2$ & 2.46 & 2.30 & 1.42 \\
\hline & \multirow[t]{3}{*}{ Mesial (tension) } & T0 & 2.52 & 2.12 & 1.79 \\
\hline & & $\mathrm{T} 1$ & 2.95 & 2.02 & 2.42 \\
\hline & & $\mathrm{T} 2$ & 2.59 & 1.97 & 1.78 \\
\hline
\end{tabular}

T0: Baseline; T1: One hour after load application; T2: 28 days after load application; IL-6: interleukin 6, GCF: gingival crevicular fluid

\subsection{Comparison of level of IL-6 and TNFa at the mesial and distal sites of control teeth at different time points}

As shown in Tables 1 and 2, level of IL-6 (T0: $p=0.610, T 1: p=0.959, T 2: p=0.241)$ and TNF $\alpha(T 0: p=0.093, T 1$ : $\mathrm{p}=0.799, \mathrm{~T} 2: \mathrm{p}=0.959)$ at both mesial and distal sites of control teeth at $\mathrm{T} 1$ and $\mathrm{T} 2$ remained the same as baseline (T0). Wilcoxon test found no significant difference at the mesial and distal sites of the control teeth at the designated time points.

\subsection{Comparison of level of IL-6 and TNFa at the mesial and distal sites of study teeth at different time points}

As shown in Tables 1 and 2, the level of cytokines at T1 was higher than that at T2. At T2, level of IL-6 (T0: $\mathrm{p}=0.445, \mathrm{~T} 1: \mathrm{p}=0.515, \mathrm{~T} 2: \mathrm{p}=0.646)$ and TNF $\alpha(\mathrm{T} 0: \mathrm{p}=0.959, \mathrm{~T} 1: \mathrm{p}=0.508, \mathrm{~T} 2: \mathrm{p}=0.263)$ decreased to the baseline value. However, the Friedman test found no significant difference among the three time points in this regard.

\subsection{Comparison of level of IL-6 and TNFa in tension and compression sites of study teeth}

Increase in level of both cytokines at the tension side compared to the pressure side at T1 in comparison to T2 is shown in Tables 1 and 2, respectively. The level of IL-6 (0.610) and TNF $\alpha(p=0.508)$ was higher at the tension side 
compared to the pressure side; however, Wilcoxon test revealed no significant difference between the two sides in test teeth.

\subsection{Comparison of changes in level of IL-6 and TNFa in the study and control teeth}

The mean changes in IL-6 $(\mathrm{p}=0.508)$ and $\mathrm{TNF} \alpha(\mathrm{p}=0.508)$ at $\mathrm{T} 1$ at both sides of the study teeth were greater than those at both sides of the control teeth; although the differences were not statistically significant according to the Wilcoxon test.

Table 2. Level of TNF $\alpha$ cytokine in GCF of test and control canine teeth (in pictograms per microliter)

\begin{tabular}{|c|c|c|c|c|c|}
\hline Group & Side & Measurement time & Mean & Standard deviation & Median \\
\hline \multirow[t]{6}{*}{ Test } & \multirow[t]{3}{*}{ Distal (pressure) } & T0 & 3.66 & 2.28 & 2.86 \\
\hline & & $\mathrm{T} 1$ & 6.38 & 6.01 & 3.71 \\
\hline & & $\mathrm{T} 2$ & 5.19 & 2.26 & 5.72 \\
\hline & \multirow[t]{3}{*}{ Mesial (tension) } & T0 & 4.14 & 2.95 & 3.16 \\
\hline & & $\mathrm{T} 1$ & 7.12 & 5.42 & 6.27 \\
\hline & & $\mathrm{T} 2$ & 4.26 & 2.30 & 3.52 \\
\hline \multirow[t]{6}{*}{ Control } & \multirow[t]{3}{*}{ Distal (pressure) } & $\mathrm{T} 0$ & 3.00 & 1.86 & 2.22 \\
\hline & & $\mathrm{T} 1$ & 4.23 & 3.21 & 3.45 \\
\hline & & $\mathrm{T} 2$ & 4.11 & 1.77 & 4.22 \\
\hline & \multirow[t]{3}{*}{ Mesial (tension) } & $\mathrm{T} 0$ & 4.80 & 2.45 & 4.71 \\
\hline & & $\mathrm{T} 1$ & 4.06 & 2.21 & 3.64 \\
\hline & & $\mathrm{T} 2$ & 3.92 & 1.98 & 3.65 \\
\hline
\end{tabular}

T0: Baseline; T1: One hour after load application; T2: 28 days after load application;

TNF $\alpha$ : tumor necrosis factor alpha, GCF: gingival crevicular fluid

\section{Discussion}

In our study, level of IL-6 and TNF $\alpha$ remained at the baseline level at all time points and level of these two cytokines did not change significantly. This finding was in agreement with that of Karaduman and Uematsu (4, 20), in that they showed no change in the level of IL-6 and TNF $\alpha$ in the control group during orthodontic treatment. Insignificant change in the level of inflammatory cytokines at the control side indicates that these markers only express when significant changes in bone metabolism occur, such as orthodontic force application, which induces periodontal changes by involving an inflammatory response (21). In our study, the level of IL-6 and TNF $\alpha$ at both mesial and distal sides of the study teeth was higher than the baseline values at one hour after load application. At T2, the level of both cytokines decreased almost to the baseline level; but the difference in this regard between different time points was not significant. This finding was in accordance with that of Basaran (22) and Ozcun (23) who reported no significant alterations in the level of pro-inflammatory cytokines following initiation of orthodontic treatment. However, in the study by Kaya et al., levels of IL- 6 and TNF $\alpha$ reached their maximum at three days in rats and then started to decrease from days seven and 10 onwards (18). Ren et al. showed that the level of IL-6 and $\mathrm{TNF} \alpha$ significantly increased at 24 hours, and during the linear phase of OTM, concentration of these cytokines decreased almost to the baseline level (24). Differences between our results and those of previous studies may be attributed to different assessment time points and variability in the magnitude of mechanical loads applied, because different magnitudes of tensile load cause different responses in the PDL (25), also, variability in the level of proinflammatory mediators is related to the type of load applied and frequency of load activation. Although the level of mediators increases following application of both continuous and interrupted loads, greater level of inflammation is seen following the application of interrupted load (26). Since continuous load was applied in our study, insignificant increase in the level of pro-inflammatory cytokines is justified. Also, insignificant change in the level of TNF $\alpha$ in our study may be due to the synergistic effects of cytokines on one another, which prevent significant increase in the level of other cytokines (27).

In our study, the level of IL-6 and TNF $\alpha$ at both sides of the study teeth was higher than that in the control teeth at $\mathrm{T} 1$, but this difference was not statistically significant. The results of this study are inconsistent with previous studies such as Karaduman et al., who showed that the concentration of TNF $\alpha$ at both sides of test teeth on days one and seven was significantly higher than that in control teeth (4) In the study by Uematsu, the level of IL-6 and TNF $\alpha$ at 24 hours after load application around test teeth was much higher than that around control teeth (20). In the study by Lowney et al., the level of $\mathrm{TNF} \alpha$ after load application was two times higher than that in control teeth (teeth before load application) (28). 
Considering the reported peak values at 24 hours in most studies such as those of Uematus et al., (20) Lee et al., (29) Ren et al., (24) and Karaduman et al. (4), differences between our results and those of previous studies may be attributed to the time-dependent concentration of pro-inflammatory cytokines (21). Regarding the levels of cytokine in GCF over time, the most commonly reported results were peak levels of cytokines at one day after orthodontic force, regardless of treatment mechanics (30), thus a one-hour time period after load application is not long enough for release of a significant amount of pro-inflammatory cytokines into the GCF, and insignificant change in the level of these cytokines at one hour was expected in our study. On the other hand, insignificant increase in level of these cytokines in study teeth (compared to controls) at one hour (compared to baseline) indicates that chemotactic activity rapidly increases after mechanical load application (17). On the other hand, increase in level of these cytokines in test teeth (compared to controls) at one hour (compared to baseline) indicates increased inflammation following mechanical stress; although it was not statistically significant.

In our study, the level of IL-6 and TNF $\alpha$ at one hour after load application was higher in the tension (mesial) side compared to the pressure side in the test teeth, although this difference was not significant. In the study by grant et al., the level of IL-6 and TNF $\alpha$ at four hours following orthodontic load application in the tension side was significantly higher than that in the pressure side (5). The results of this study are similar to those of Dudic et al. (31). The results of this study contradict previous studies such as Karaduman, Garlet, Kaya, and Blesta (4, 16, 32, 33). These studies showed that the concentration of pro-inflammatory cytokines at the pressure site was higher than the tension site. The controversy in our results and those of previous studies may be explained by the fact that roots undergoing OTM have different anatomical shapes and morphology and we cannot consider a specific geometry for all teeth. Load applied to teeth causes biological response in the entire periodontal structure and the GCF freely circulates in the entire gingival sulcus. Finite Element Analysis shows that the compression and tension areas can be seen only when the PDL is modeled as a linear material. It can only be concluded that cytokine levels in GCF may not be indicative of periodontal remodeling in pressure and tension. It has been suggested that the cellular response may be part of the overall increase in metabolic activity, not responding to mechanical stress (30). Thus, the level of cytokines cannot be well differentiated at the pressure and tension sites, and collection of GCF from the mesial and distal sides alone cannot serve as a suitable indictor for assessment of activity at the pressure and tension sites. The limitations of this investigation include low sample size and no use of the periotron device to determine the volume of collected GCF.

\section{Conclusions}

Immediate increase (although insignificant) in the level of pro-inflammatory cytokines at one hour after mechanical stress (load) application to teeth, highlights the significant role of these cytokines in the process of periodontal tissue remodeling. This supports the important role of cytokines in OTM. Since load applied to teeth causes biological response in the entire periodontal structure, and GCF freely circulates in the entire gingival sulcus, the level of mediators in the collected GCF from the mesial and distal sides cannot serve as a suitable indicator for evaluation of activity at the pressure and tension sites.

\section{Acknowledgments:}

The manuscript is an original work of authors, based on a post graduate degree of Orthodontics (Ref. No.: 40). Many thanks to the Immunology Department of Qazvin University of Medical Science for assessment of cytokines level and the Orthodontics Department of Qazvin Faculty of Dentistry, which provided the necessary orthodontics materials, and the cooperation of patients participating in this research. Also thanks to the financial support of Qazvin University of Medical Sciences.

\section{Trial registration:}

The trial was registered at the Iranian Registry of Clinical Trials with the IRCT ID: IRCT2017030632903N2.

\section{Funding:}

The present study was supported by a grant from the Research Council, Qazvin University of Medical Sciences, Qazvin, Iran (Ref: 40).

\section{Conflict of Interest:}

There is no conflict of interest to be declared. 
Authors' contributions:

All authors contributed to this project and article equally. All authors read and approved the final manuscript.

\section{References:}

1) Al-Ghurabi BH. Mohammed-Salih HS, Ghazi A, Saloom HF. Evaluation of Salivary Levels of Proinflammatory Cytokines (IL1 $\alpha$, IL8 and GM-CSF) in Adult Orthodontic Patients. Journal of Dental and Medical Sciences. 2014; 13(3): 75-8.

2) Kitaura H, Kimura K, Ishida M, Sugisawa H, Kohara H, Yoshimatsu M, et al. Effect of Cytokines on Osteoclast Formation and Bone Resorption during Mechanical Force Loading of the Periodontal Membrane. Scientific World Journal. 2014; 2014: 617032. doi: 10.1155/2014/617032. PMID: 24574904, PMCID: PMC3916098.

3) Jiang C, Li Z, Quan H, Xiao L, Zhao J, Jiang C, et al. Osteoimmunology in orthodontic tooth movement. Correction(s) for this article. Oral Dis. 2015; 21(6): 694-704. doi: 10.1111/odi.12273. PMID: 25040955.

4) Karaduman B, Uraz A, Altan GN, Tuncer BB, Alkan Ö, Gönen S, et al. Changes of tumor necrosis factor$\alpha$, interleukin-10, and tartrate-resistant acidphosphatase $5 \mathrm{~b}$ in the crevicular fluid in relation to orthodontic movement. European Journal of Inflammation.2015; 13(1): 3-13. doi: 10.1177/1721727X15573917.

5) Grant M, Wilson J, Rock P, Chapple I. Induction of cytokines, MMP9, TIMPs, RANKL and OPG during orthodontic tooth movement. European Journal of Orthodontics. 2013; 35(5): 644-5. doi: 10.1093/ejo/cjs057. PMID: 22987319.

6) Pramustika A, Soedarsono N, Krisnawati, Widayati R. Comparison of Tumor Necrosis Factor- $\alpha$ Concentrations in Gingival Crevicular Fluid between Self-Ligating and Preadjusted Edgewise Appliances in the Early Leveling Stage of Orthodontic Treatment. Contemp Clin Dent. 2018; 9(1): 92-6. doi: 10.4103/ccd.ccd_792_17. PMID: 29599592, PMCID: PMC5863418.

7) $\mathrm{Hu} \mathrm{Y,} \mathrm{Liu} \mathrm{W,} \mathrm{Liu} \mathrm{Z,} \mathrm{Kuang} \mathrm{W,} \mathrm{He} \mathrm{H.} \mathrm{Receptor} \mathrm{activator} \mathrm{of} \mathrm{nuclear} \mathrm{factor-kappa} \mathrm{ligand,} \mathrm{OPG,} \mathrm{and} \mathrm{IGF-I}$ expression during orthodontically induced inflammatory root resorption in the recombinant human growth hormone-treated rats. Angle Orthod. 2015; 85(4): 562-9. doi: 10.2319/052014-361.1. PMID: 25184787.

8) Kunii R, Yamaguchi M, Tanimoto Y, Asano M, Yamada K, Goseki T, et al. Role of interleukin-6 in orthodontically induced inflammatory root resorption in humans. The Korean Association of Orthodontists. 2013; 43(6): 294-301.

9) Scheller J, Chalaris A, Schmidt-Arras D, Rose-John S. The pro- and anti-inflammatory properties of the cytokine interleukin-6. Biochim Biophys Acta. 2011; 1813(5):878-88. doi: 10.1016/j.bbamcr.2011.01.034. PMID: 21296109.

10) Guo S, Ni Y, Ben J, Xia Y, Zhou T, Wang D, et al. Class A Scavenger Receptor Exacerbates Osteoclastogenesis by an Interleukin-6-Mediated Mechanism through ERK and JNK Signaling Pathways. Int J Biol Sci. 2016; 12(10): 1155-67. doi: 10.7150/ijbs.14654. PMID: 27766031, PMCID: PMC5069438.

11) Alves ACA. The impact of orthodontic treatment on periodontal support loss. Dental Press J Orthod. 2012; 17(1): 18-20. doi: 10.1590/S2176-94512012000100002.

12) d'Apuzzo F, Cappabianca S, Ciavarella D, Monsurrò A, Silvestrini-Biavati A, Perillo L. Biomarkers of Periodontal Tissue Remodeling during Orthodontic Tooth Movement in Mice and Men: Overview and Clinical Relevance. The Scientific World Journal. 2013; 2013(1): 1-8. doi: 10.1155/2013/105873. PMID: 23737704, PMCID: PMC3655650.

13) MacLaine JK, Rabie AB, Wong R. Does orthodontic tooth movement cause an elevation in systemic inflammatory markers? European Journal of Orthodontics. 2010; 32(4): 435-40. doi: 10.1093/ejo/cjp108. PMID: 19822530.

14) Kojima T, Yamaguchi M, Yoshino T, M Shimizu, Yamada K, Goseki T, et al. TNF- $\alpha$ and RANKL facilitates the development of orthodontically-induced inflammatory root resorption. Open Journal of Stomatology. 2013; 3: 52-8.

15) de Aguiar MC, Perinetti G, Capelli J Jr. The Gingival Crevicular Fluid as a Source of Biomarkers to Enhance Efficiency of Orthodontic and Functional Treatment of Growing Patients. Biomed Res Int. 2017; 2017: 3257235. doi: 10.1155/2017/3257235. PMID: 28232938, PMCID: PMC5292379.

16) Garlet TP, Coelho U, Silva JS, Garlet GP. Cytokine expression pattern in compression and tension sides of the periodontal ligament during orthodontic tooth movement in humans. Eur J Oral Sci. 2007; 115(5): 355362. doi: 10.1111/j.1600-0722.2007.00469.x. PMID: 17850423. 
17) Zainal Ariffin SH, Yamamoto Z, Zainol Abidin IZ, Megat Abdul Wahab R, Zainal Ariffin Z. Cellular and molecular changes in orthodontic tooth movement. Scientific World Journal. 2011; 2011(11): 1788-803. doi: 10.1100/2011/761768. PMID: 22125437, PMCID: PMC3201678.

18) Acun Kaya F, Gunduz Arslan S, Kaya CA, Arslan H, Hamamci O. The Gingival Crevicular Fluid Levels of IL-1 $\beta$, IL-6 and TNF- $\alpha$ in Late Adult Rats. Int Dent Res 2011; 1(1): 7-12. doi: 10.5577/intdentres.2011.vol1.no1.2.

19) Alikhani M, Alyami B, Lee IS, Almoammar S, Vongthongleur T, Alikhani M, et al. Saturation of the response to orthodontic forces biological and its effect on the rate of tooth movement. Orthod Craniofac Res. 2015; 18(1): 8-17. doi: 10.1111/ocr.12090. PMID: 25865529.

20) Uematsu S, Mogi M, Deguch T. Levels Are Elevated Interleukin (IL)-1b, IL-6, Tumor Necrosis Factor- $\alpha$, Epidermal Growth Factor, and $\beta 2$-Microglobulin in Gingival Crevicular Fluid during Human Orthodontic Tooth Movement. J Dentt Res. 1996; 75(1): 562-7. doi: 10.1177/00220345960750010801. PMID: 8655760.

21) Ren Y, Vissink A. Cytokines in crevicular fluid and orthodontic tooth movement. Eur J Oral Sci. 2008; 116(2): 89-97. doi: 10.1111/j.1600-0722.2007.00511.x. PMID: 18353001.

22) Basaran G, Özer T, Acun Kaya F, Hamamci O. Interleukins 2, 6, and 8 levels in human gingival sulcus during orthodontic treatment. Am J Orthod Dentofacial Orthop. 2006; 130(1): 7. e1 -6. PMID: 16849065.

23) Özcan SS, Ceylan I, Özcan E, Kurt N, Dağsuyu IM, Çanakçi CF. Evaluation of Oxidative Stress Biomarkers in Patients with Fixed Orthodontic Appliances. Journal of Desease markers. 2014; 2014 (597892): 1-6.

24) Ren Y, Hazemeijer H, de Haan B, Qu N, de Vos P. Cytokine profiles in crevicular fluid during orthodontic tooth movement of short and long durations. J Periodontol. 2007; 78(3): 453-8. doi: 10.1902/jop.2007.060261. PMID: 17335368.

25) Karacay S, Saygun I, Bengi AO, Serdar M. Tumor necrosis factor-alpha levels during two different canine distalization techniques. Angle Orthod. 2007; 77(1): 142-7. doi: 10.2319/120905-430R.1. PMID: 17029549.

26) Kapoor P, Kharbanda OP, Monga N, Miglani R, Kapila S. Effect of orthodontic forces on cytokine and receptor levels in gingival crevicular fluid: a systematic review. Prog Orthod. 2014; 15(65): 1-21. doi: 10.1186/s40510-014-0065-6.

27) Saito S, Ngan P, Saito M, Lanese R, Shanfeld J, Davidovitch Z. Interactive effects between cytokines on PGE production by human periodontal ligament fibroblasts in vitro. J Dent Res. 1990; 69(8): 1456-62. doi: 10.1177/00220345900690080201. PMID: 2117029.

28) Lowney JJ, Norton LA, Shafer DM, Rossomando EF. Orthodontic forces increase tumor necrosis factor $\alpha$ in the human gingival sulcus. Am J Orthod Dentofacial Orthop. 1995; 108(5): 519-24. doi: 10.1016/S08895406(95)70052-8.

29) Lee KJ, Park YC, Yu HS, Choi SH, Yoo YJ. Effects of continuous and interrupted orthodontic force on interleukin-1 $\beta$ and prostaglandin $\mathrm{E} 2$ production in gingival crevicular fluid. American Journal of Orthodontics and Dentofacial Orthopedics. 2004; 125(2): 168-77. doi: 10.1016/j.ajodo.2003.03.006. PMID: 14765054.

30) Allgayer S, de Menezes LM, Lemos Rinaldi MR. Cytokines in crevicular fluid and orthodontic force: a systematic review. Rev Odonto Cienc. 2017; 32(2): 104-10. doi: 10.15448/1980-6523.2017.2.26330.

31) Dudic A, Kiliaridis S, Mombelli A, Giannopoulou C. Composition changes in gingival crevicular fluid during orthodontic tooth movement: comparisons between tension and compression sides. European Journal of Oral Sciences. 2006; 114(5): 416-22. doi: 10.1111/j.1600-0722.2006.00387.x. PMID: 17026508.

32) Kaya FA, Hamamci N, Basaran G, Dogru M, Yildirim TT. TNF- $\alpha$, IL-1 $\beta$ and IL-8 levels in tooth early leveling movment orthodontic treatment. Journal of International Dental And Medical Research. 2010; 3(3): 116-21.

33) Bletsa A, Berggreen E, Brudvik P. Interleukin-1a and tumor necrosis factor-a expressionduring the early phases of orthodontic tooth movement in rats. Eur J Oral Sci. 2006; 114(5): 423-9. doi: 10.1111/j.16000722.2006.00400.x. PMID: 17026509. 